\title{
LASER OPTOGALVANIC SPECTROSCOPY OF MOLECULES: THE RED ELECTRONIC SYSTEM OF TITANIUM OXIDE (TIO)
}

\author{
Ronald C. CARLSON ${ }^{1}$, Lee A. CROSS ${ }^{2}$ and Thomas M. DUNN \\ Department of Chemistry. The University of Michugan, Ann Arbor, Michigan 48109. USA
}

Received 26 July 1984; in final form 6 October 1984

\begin{abstract}
The laser optogalvanic (LOG) spectrum of the $(0,0)$ bands of the $B^{3} \Pi-X^{3} \Delta$ transition of titanium, oxide (T1O) has been observed. The TjO molecules were generated in a glow discharge by sputtering titanium metal and $\mathrm{TiO}_{2}$ from the inner surface of a hollow cathode. Possible mechanisms for the molecular LOG effect are discussed
\end{abstract}

\section{Introduction}

The laser optogalvanic (LOG) effect has found numerous applications in gas-phase spectroscopy since the first experiments by Green and co-workers were reported in 1976 [1]. The LOG phenomenon is the change which is produced in the conductivity of a flame or discharge when radiation from a tunable laser causes a constituent species to undergo transitions to higher-lying electronic or vibrational states. The spectrum which is recorded is the change in the conductivity of the medium as a function of the wavelength of the incident radiation. One of the most exciting and useful features of LOG spectroscopy is its ability to detect transitions in species such as neutral and charged radicals which are difficult to observe by other methods. The LOG technique also makes it possible to detect transitjons which originate from highly excited energy states which are frequently not populated in emission, absorption, or laser-induced fluorescence studies. This is due in part to the fact that the sources employed for LOG experuments - flames and electrical discharges - are character-

1 Current address. Department of Chemistry, University of Califomia, and Materials and Molecular Research Division, Lawrence Berkeley Laboratory, Berkeley, Calf̈fornia 94720 , USA.

2 Current address: University of Dayton Research Institute, 300 College Park Avenue, Dayton, Ohio 45469, USA. szed by a high degree of electronic excitation of neutral and ionic species. As the present study will illustrate shortly, LOG spectroscopy therefore offers the powerful ability to simplufy an emission or absorption spectrum by selectively favoring some transitions over others.

Although the first LOG studies were confined to atomic spectroscopy, a number of molecular spectra in both the visible and infrared have been reported recently [2-5]. The purpose of the present research was to detect optogalvanic signals from transition metal diatomic molecules generated un situ in nonflame media. We were successful in obtaining $L O G$ spectra of the $(0,0)$ bands of the $B{ }^{3} \Pi \leftarrow X^{3} \Delta$ transition of titanum oxide ( $\mathrm{TiO}$ ). The TiO molecules were generated in a glow discharge by sputtering titanium metal and $\mathrm{TiO}_{2}$ from the inner surface of a hollow cathode composed of a hughly compressed mixture of these two substances. Argon was used as the diccharge-supportung gas and sputtering agent.

\section{Experimental}

The experimental arrangement is shown in fig. 1 . The incident laser beam is directed along the axis of the hollow cathode, thereby covering the front surface of the anode (see fig. 3 below). The laser dye used was rhodamine $6 \mathrm{G}$ in $\mathrm{H}_{2} \mathrm{O} / 4 \%$ amonyx-LO solvent. The radiation first passes through a Corning 


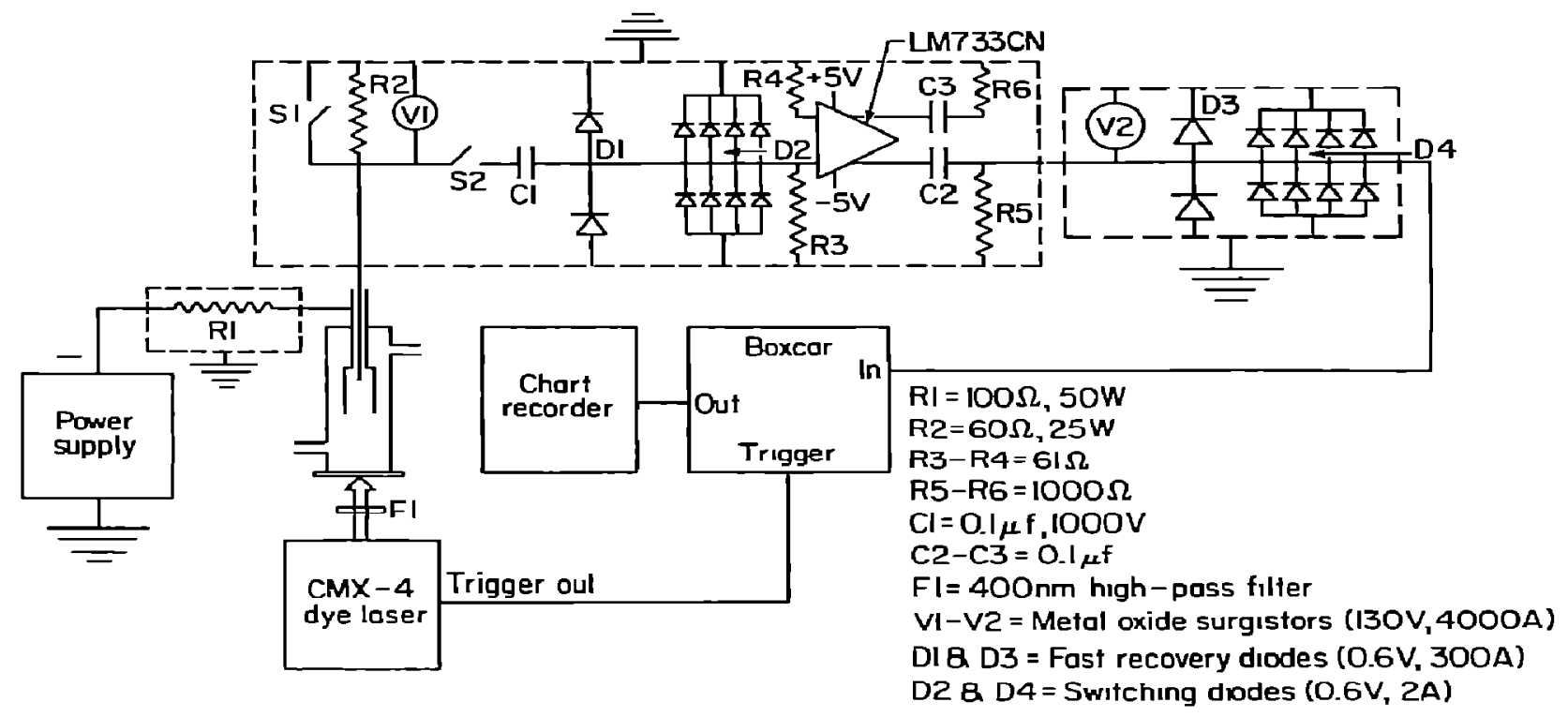

Fig 1. Experimental arrangement.

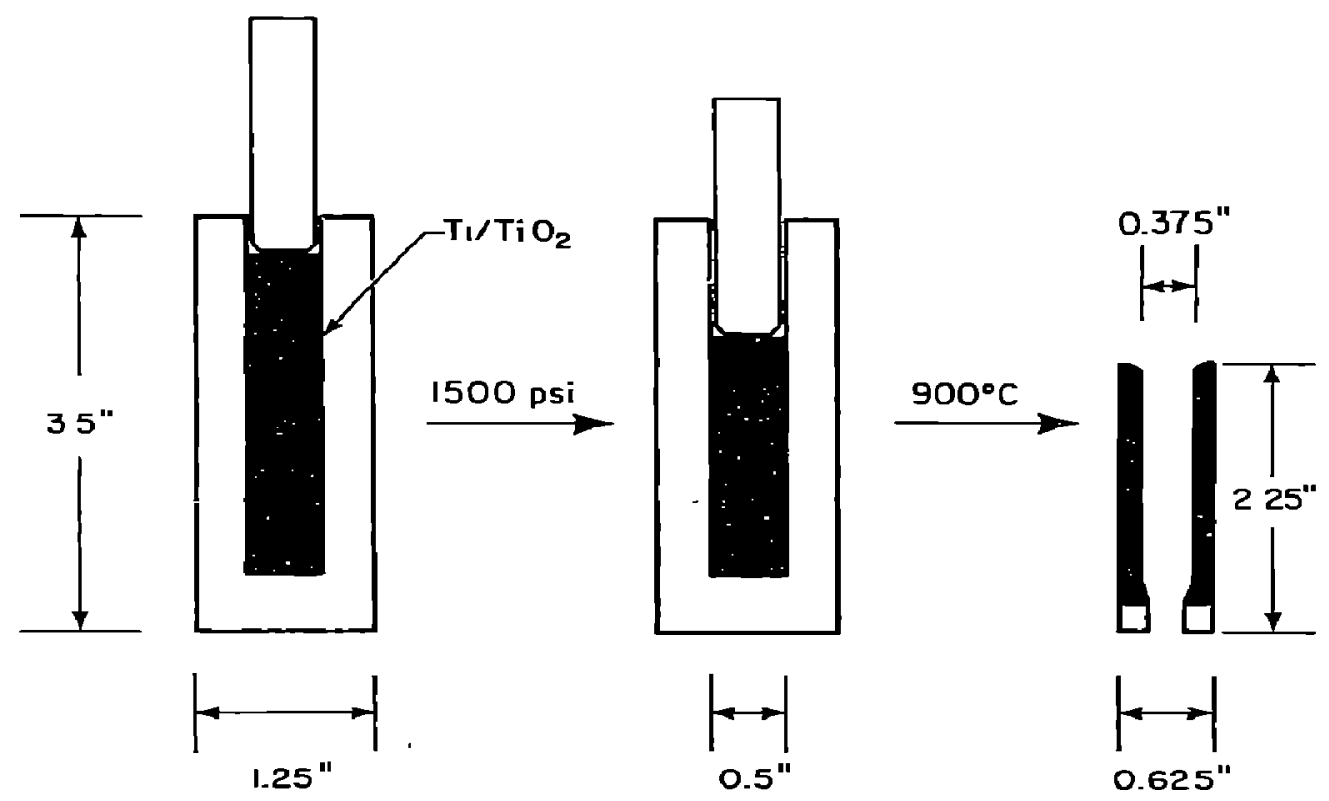

Fig. 2. Construction of the hollow cathode. 
CS 0-51 filter, which cuts off sharply below $400 \mathrm{~nm}$. The dotted lines in fig. 1 represent rf-shielded enclosures. (The $60 \Omega$ load resistor and the amplifier were later replaced by a $4500 \Omega, 480 \mathrm{~W}$ load resistor.) The protective networks $\left(V_{1}, D_{1}, D_{2}\right)$ and $\left(V_{2}, D_{3}, D_{4}\right)$ were added to the circuit to shield the amplifier and the boxcar averager, respectively, from hazardous current transients which occasionally arise from the discharge cell.

The construction of the hollow cathode is illustrated in fig 2. A mixture of $\mathrm{TiO}_{2}$ and $\mathrm{Ti}$ (325 mesh, source: Alfa Division, Ventron Corp) in the molar ratio of $0.34 / 100$ was compressed to 1500 psi in a steel cylinder. After two days at this pressure, the cylinder was slowly rassed to $900^{\circ} \mathrm{C}$ and was maintanned at this temperature for $24 \mathrm{~h}$. Upon cooling, the cylinder was machined to form the finished hollow cathode. All surfaces were highly polished in order to prevent spurious arcing.

Fig. 3 is a diagram of the glow discharge cell assembly. The hollow cathode is connected to the negative high-voltage terminal of the power supply via the stamless steel tube. The cathode is insulated from the stainless steel anode rod by an aluminum oxide tube. As is seen from the diagram, the anode is inside the hollow cathode and along its axis. A fused silica discharge shield covers all but the front $4 \mathrm{~mm}$ of the cathode.

Argon is continuously flowed through the cell at a pressure of $\approx 0.5$ Torr: Argon ions sputter $\mathrm{TiO}_{2}$ and Ti from the inner surface of the cathode, and the ensung reaction forms $\mathrm{TiO}$. It is well known that sputtering efficiency increases with decreasing pressure of the sputtering gas, but it was found that the discharge would often become noisy or extunguish itself if the argon pressure were much lower than 0.5 Torr. Sputtering efficiency also increases with atomic weight of the sputtering gas; hence argon was used in lieu of helium or neon. In fact, no optogalvanic sigizals from TiO were observed when helium was substituted for argon $\mathrm{A}$ torus of bright red-orange TiO emission of $\approx 1 \mathrm{~mm}$ thickness was observed on the inner surface of the cathode. Further towards the axis only the blue glow of argon could be seen.

As a preliminary to the LOG experiments, emission spectra of the discharge were photographed using a $15 \mathrm{~m}$ grating spectrograph with a resolving power of 35000 in the first order. Kodak 103 a-F film was used, with a slit width of $10 \mu \mathrm{m}$ and exposure times of 4-6 min. Spectra were photographed using discharge cur-

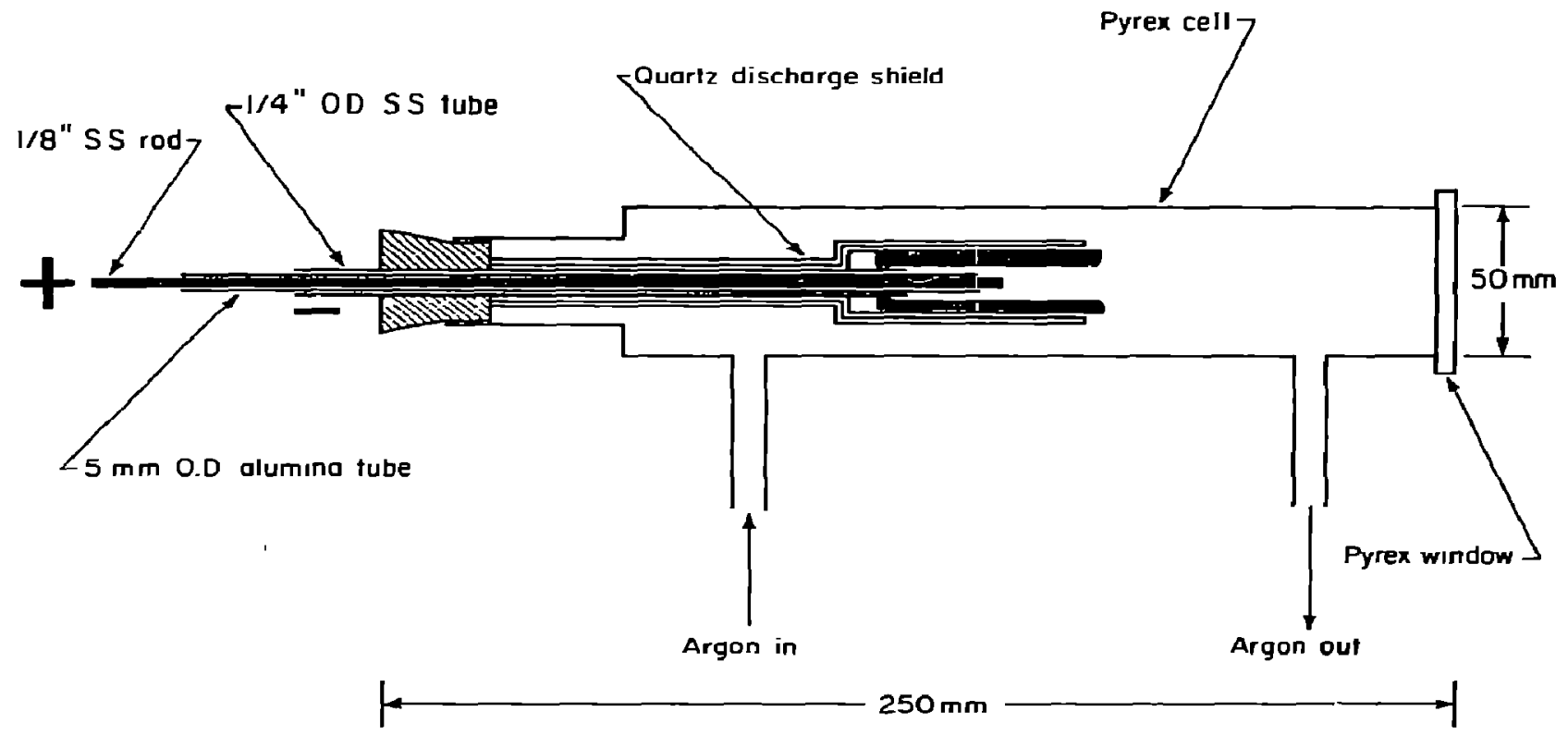

Fig- 3. Glow discharge cell assembly. 
rents of 50-100 $\mathrm{mA}$ and argon pressures of $0.5-0.8$

Torr. Every spectrum showed strong TiO emission from the three allowed components of the $B^{3} \Pi \leftarrow$ $X^{3} \Delta$ system, which extends from $\approx 614$ to $\approx 628 \mathrm{~nm}$ [6-9]. Because the TiO features were present to such a pronounced degree in all of the emission spectra, it was decided to restrict the discharge currents for the LOG experiments to $60-90 \mathrm{~mA}$. It was observed that a cathode had to be run for at least two hours in the discharge before TiO emission began to appear.

\section{Wavelength calibration}

In order to calibrate molecular LOG spectra for wavelength, advantage was taken of the fact that each molecular spectrum included an LOG spectrum of argon, suce the latter was the discharge-supporting gas The argon transitions were identified by recording simultaneous LOG spectra of argon and neon. This was accomplished by using a beam splitter to direct a fraction of the laser beam into a commercial $\mathrm{Ne} / \mathrm{Fe}$ hollow cathode lamp. Since the optogalvanic spectrum of neon in the rhodamine $6 \mathrm{G}$ wavelength region is well known (refs. [10-14] and present work), the argon transitions could be easily matched with their optical counterparts [15]. (There were, in fact, no LOG argon transitions which did not correspond to the transitions in ref [15].) A molecular spectrum was then calibrated for wavelength by fitting the frequencies of its argon transitions to a second-degree polynomial with respect to their positions on the chart recording.

\section{Results and discussion}

Fig. 4 shows LOG spectra of the $B-X$ system of $\mathrm{T} 1 \mathrm{O}$ recorded using two different $\mathrm{T} 1 / \mathrm{TiO}_{2}$ hollow cathodes. Above them is a low-resolution emission spectrum of TiO [6], and at the bottom is an optogalvanic spectrum of argon taken using a freshly made hollow cathode from which TiO emission had not yet appeared. What is plotted in the LOG spectra is the potential difference across the load resstor, R2, as a function of the wavelength of the incident laser light. The argon spectrum clearly exhibits no features at the wavelengths of the TiO band heads other than the ex-

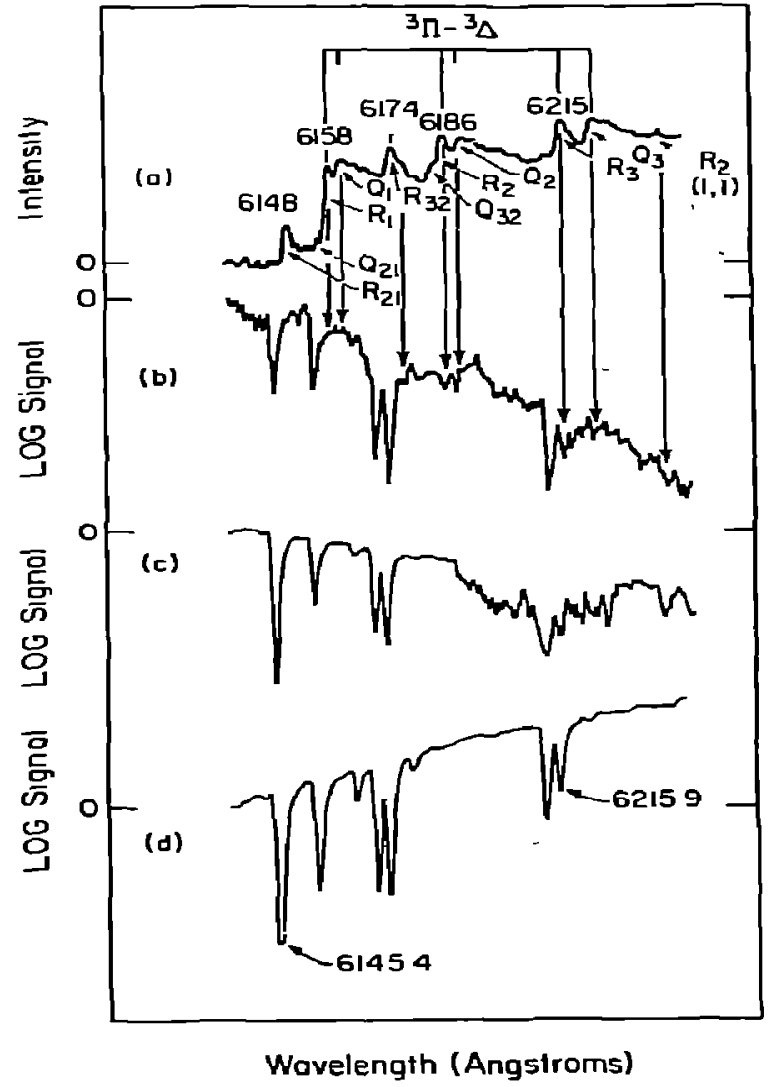

Fig- 4. (a) Low-resolution emission spectrum of TiO, $T=$ $2100^{\circ} \mathrm{C}[6]-(b, c)$ LOG spectra of TiO taken using two dufferent $\mathrm{T} 1 / \mathrm{TiO}_{2}$ hollow cathodes. (d) LOG spectrum of argon taken using a freshly made $\mathrm{Ti} / \mathrm{TiO}_{2}$ hollow cathode from which no T1O emission was observed on the photographic film.

pected Ar I transitions [15]. The individual "lines" observed in the TiO spectra are red-degraded due to the nature of the molecular band profiles and not to a time constant effect during the recording.

All of the LOG transitions in fig. 4 correspond to negative changes in voltage across $\mathrm{R} 2$. It is therefore apparent from fig. 1 that the laser-induced transitions between energy states of both TiO and argon produce an increase in the conductivity of the medium, i.e. an increase in the current through the discharge cell The most likely mechanism for the TiO transitions is deexcitation of the TiO(B ${ }^{3} \Pi$ ) by superelastic collisions with slow electrons in the hollow cathode [3]:

$e^{-}($slow $)+\operatorname{TiO}_{1}\left(B^{3} \Pi\right) \rightarrow e^{-}$(fast) $\left.+\operatorname{TiO}^{3} X^{3} \Delta\right)$. 
Another possible mechanism, laser-enhanced ionuzation, is certainly important in the case of the argon transitions, which depend strongly upon the existence of high-iying metastable states of $\operatorname{Ar} I[13,14$, 16] Enhanced ionization is not in this case, however, expected to contribute significantly to the production of fast electrons following laser excitation of the $\mathrm{TiO}$ molecules. We know that for TiO, the energy difference between the $B^{3} \Pi$ and $X^{3} \Delta$ states is $\approx 2 \mathrm{eV}$. On the other hand, the ionization potential of TiO is $\approx 5.5 \mathrm{eV}$ [17]. Therefore, we do not expect much of an increase in the rate of ionization of the TiO molecules to follow laser excitation to the ${ }^{3} \Pi$ state.

Although the $(0,0)$ bands of the $B-X$ system of TiO are observed in both the emission and the LOG spectra, the present example clearly illustrates the power of LOG spectroscopy to selectively favor certain transitions. Of the three principal sub-bands of the B-X system, that of lowest energy, ${ }^{3} \Pi_{0} \leftarrow$ ${ }^{3} \Delta_{1}$, is the least intense in both the emission and the LOG spectra. It is relatively weaker in the latter, however, since the hollow cathode discharge strongly disfavors the population of the ${ }^{3} \Delta_{1}$ substrate. It is also of interest to note that the sub-band of intermediate energy, ${ }^{3} \Pi_{1} \leftarrow{ }^{3} \Delta_{2}$, s relatively less intense in fig. $4 b$ than in fig. 4c. The difference is not extreme and can most likely be attributed to the difficulty of precisely reproducing the conditions of a hollow cathode discharge of the present type. One can also see that the $R_{2}(1,1)$ transition is more prominent in the LOG spectra than in the emission spectrum. On the basis of the preceding discussion it is anticipated that future LOG studies will succeed in observing molecular energy states which have heretofore escaped detection.

\section{Acknowledgement}

The authors gratefully acknowledge discussion of this work with Professor Michael D. Morns, University of Michigan, Mr. Charles Stout, Environmental Research Institute of Michigan, Ann Arbor, and Professor Richard J. Saykally, University of Califomia, Berkeley.

\section{References}

[1] R.B. Green, R.A. Keller, G.G. Luther, P.K. Schenck and J_C. Travis, Appl Phys. Letters 29 (1976) 727.

[2] C.R. Webster and C T. Rettner, Laser Focus 19 (1983) 41, and references therein.

[3] J. Pfaff, M.H. Begemann and R.J. Saykally, Mol. Phys. 11 (1984) 1.

[4] R. Walkup, R.W. Dreyfus and P. Avouris, Phys. Rev. Letters 50 (1983) 1846.

[5] R. Klein, R.P. McGinnis and S.R. Leone, Chem. Phys. Letters 100 (1983) 475.

[6] J.G Phillips, Astrophys. J. 169 (1971) 185.

[7] J.G Phillips, Astrophys. J. Suppl. Series No. 232, 26 (1973) 313

[8] K.P. Huber and G. Herzberg, Molecular spectra and molecular structure, Vol. 4. Constants of diatomic molecules (Van Nostrand, Princeton, 1979).

[9] W.H. Hocking, M.C.L. Gerry and A.J. Merer, Can. J. Phys. 57 (1979) 54.

[10] D.S. King, P.K. Schenck, K.C. Smyth and J C. Travs, Appl. Opt. 16 (1977) 2617.

[11] K C. Smyth and P.K. Schenck, Chem. Phys. Letters 55 (1978) 466

[12] W.M. Bridges, J. Opt. Soc. Am. 68 (1978) 352.

[13] K C. Smyth, R.A. Keller and F.F. Crim, Chem. Phys. Letters 55 (1978) 473

[14] E.F. Zalewski, R.A. Keller and R. Engleman Jr., J. Chem. Phys 70 (1979) 1015.

[15] A R. Striganov and N.S. Sventitikit, Tables of spectral lines of neutral and ionized atoms (IFI/Plenum Press, New York, 1968).

[16] J.C. Travis, P.K Schenck, G C. Turk and W.G Mallard, Anal Chem. 51 (1979) 1516.

[17] H.M. Rosenstock, K. Draxl, B.W Steiner and J.T. Herron, J. Phys. Chem. Ref. Data 6, suppL 1 (1977). 